\title{
Preventive maintenance and replacement policies for deteriorating production systems
}

\author{
F. I. DEHAYEM NODEM ${ }^{\mathrm{a}}$, A. GHARBI ${ }^{\mathrm{b}}$ and J.-P. KENNÉ ${ }^{\mathrm{a}}$ \\ École de technologie supérieure, 1100 Notre Dame Street West, Montréal, Que. Canada H3C 1K3 \\ a Mechanical Engineering Department, Production Technologies Integration Laboratory \\ ${ }^{\mathrm{b}}$ Automated Production Engineering Department, Production System Design and Control Laboratory \\ subject to imperfect repairs
}

\begin{abstract}
This paper presents a special case of integration of the preventive maintenance into the repair/replacement policy of a failure-prone system. The machine of the considered system exhibits increasing failure intensity and increasing repair times. To reduce the failure rate and subsequent repair times following a failure, there is an incentive to perform preventive maintenance on the machine before failure. When a failure occurs, the machine can be repaired or replaced by a new one. Thus the machine's mode at any time can be classified as either operating, in repair, in replacement or in preventive maintenance. The decision variables of the system are the repair/replacement switching age or number of failures at the time of the machine's failure and the preventive maintenance rate. The problem of determining the repair/replacement and preventive maintenance policies is formulated as a semi-Markov decision process and numerical methods are given in order to compute optimal policies which minimize the average cost incurred by preventive maintenance, repair and replacement over an infinite planning horizon. As expected, the decisions to repair or to replace the machine upon a failure are modified by performing preventive maintenance. A numerical example is given and a sensitivity analysis is performed to illustrate the proposed approach and to show the impact of various parameters on the control policies thus obtained.
\end{abstract}

Index Terms - Manufacturing systems, numerical methods, optimal control, damage failures, replacement, repair, preventive maintenance.

\section{Introduction}

Very often, parts of industrial machines are broken or highly damaged, although the machines still functioning. A failure can consequently involve more than one component and more than one kind of component. Decision maker has to find strategy that defines replacement policies of these obsolete equipments. Most of the time, the strategy is to replace gradually the old components in a corrective way, progressively with their normal outage, but at the risk of a larger number of failures. For such a deteriorating system, it is quite reasonable to assume that the successive working times of machines will become shorter and shorter while the consecutive repair times become longer and longer (Zhang et al., 2001; Leung, 2006). Due to increasing failure rate and increasing repair times, the machine may finally be economically non-reparable after it experiences a certain number of failures. Therefore, upon a failure or when certain observed condition information is higher than a pre-set critical level, a decision must be made: whether to continue to repair the machine and face the ever-increasing repair costs, or to replace the machine with a new one.

This is an Accepted Manuscript of an article published by Taylor \& Francis in International Journal of Production Research on June 29th 2010, available online: http://wwww.tandfonline.com/10.1080/00207543.2010.492798. 
Several authors contributed in the sphere of systems that deteriorate due to effects of ageing. Among them are Nakagawa and Kijima (1989), Kenne and Gharbi (1999), Wang (2000) and Ansell et al. (2004). Those authors proposed maintenance models in order to face or to counteract ageing effects. Yuniarto and Labib (2006) suggested condition based maintenance in the case where the breakdown rarely occurs, but when it does occur, it will take a long time to repair. Wang (2002) compared and classified maintenance models with the aim to permit to decision maker to see which one is convenient to solve his specific problem. According to that classification, maintenance can be divided into two major classes: corrective maintenance that occurs when the system fails and preventive maintenance that occurs when the system is operating. Replacement is qualified as a perfect maintenance (corrective or preventive). In fact, in manufacturing environment, preventive maintenance, corrective maintenance and replacement activities take place simultaneously.

Many works deal with simultaneous repair, replacement and preventive maintenance of production systems. Although in some cases the machine is as good as new after corrective or preventive maintenance (Aven and Castro, 2008), more often, maintenances are performed on machines to improve their condition but that do not renew them. Various works considered this type of improvement by using the concept of virtual age of the machine after maintenance (Makis and Jardine, 1991; Love et al., 2000; Doyen and Gaudoin, 2004). The concept of virtual age of the machine introduced by Kijima et al. (1988) and generalized by Kijima (1989), states that the maintenance serves to reset the age of the machine, which determines the revised failure intensity. The reset age is the virtual age of the machine. Previously in 1979, Malik introduced the concept of an age reduction factor (Malik, 1979). By this concept, the machine's condition is improved through maintenance actions such as cleaning, lubrication and realignment. In Malik's model, each imperfect preventive maintenance changes the initial hazard rate value immediately following the preventive maintenance, but not all the way to zero (i.e., not new). Nguyen and Murthy (1981) considered a special case of imperfect maintenance in that, it could restore the system's effect age back to zero with a higher failure rate. Lugtigheid et al. (2008) proposed replacing failed subsystems along with some non-failed parts, to take advantage of the fact that the system has already been uninstalled and taken out of operation. This sort of maintenance brings the state of the machine to somewhere between as good as new and the condition it was in immediately before the failure or preventive maintenance. Malik (1979) proposed using expert judgment to estimate the improvement factor. According to Doyen and Gaudoin (2004), age-reduction factors can be estimated directly from the data for different types of repairs. They also provided an estimation of the reduction factor, obtained by simulation.

The above-cited authors concluded that the time required for the repair(s) and preventive maintenance is either at constant rate, negligible or not necessary to be taken into account. The assumption is not realistic for all production industry machines. Very often, failures become more severe as their number increases. The repair times thus increase with the number of failures (Leung, 2006). Many works have been performed to take into account increasing repair times of deteriorating systems. Lam (1988) considered a repair and replacement model where consecutive failure repair times of the system become longer and longer and analysed optimal replacement policies. Stanley (1993) studied a repair replacement model for a deteriorating system, considering that the magnitude of the shock at each failure and the consecutive repair times after failure constitute geometric processes and are stochastically non-decreasing. He proposed a replacement policy based on a random threshold. Zhang et al. (2001) used the concept of geometric process replacement policy of 
Lam (1988), incorporated preventive maintenance and defined the objective function termed the "cost efficiency". Wang and Zhang (2006) studied a case of deteriorating system in which preventive repair times followed a stochastically increasing geometric process and determined optimal replacement policy of the machine. Badia and Berrade (2009) assumed that the mean times of repair constituted an increasing sequence from one failure to another and developed a maintenance policy of a system subject to periodical inspections, aimed at detecting the occurrence of failures. Although those authors took into account deterioration in repair times, none of them considered reduction of repair times following maintenance.

The proposed approach consists of integrating preventive maintenance with the repair/replacement policy of a deteriorating production machine. The preventive maintenance aims at increasing the system's availability by reducing the system failure intensity and the age of the system. Moreover, the next mean repair time following a preventive maintenance is reduced with a time reduction factor, which has not yet been addressed in the literature. The approach consists of developing a semi-Markov decision model in order to determine optimal preventive maintenance, repair and replacement policies for the system. Those policies should minimize the overall incurred costs over an infinite planning horizon. Thus, the contribution of this paper is the integration of preventive maintenance to the repair/replacement determination model. We transpose the well-known age reduction and hazard rate increase factor methods to repair time reduction in order to improve the lifetime of the system.

The paper is organized as follows. In Section 2, we present the problem statement. Repair time reduction approach and illustration are given in Section 3 followed by optimal control problem and optimality conditions in section 4. Numerical methods are used in Section 5 to solve the optimality conditions obtained in Section 4. Numerical examples are presented in Section 6 with an example of implementation of the present results. A sensitivity analysis is provided to illustrate the usefulness of the proposed approach in section 7. Our conclusions are presented in Section 8 .

\section{Problem statement}

In the following subsections, we will describe the dynamic of the system and present the industrial context.

\subsection{Dynamic of the system}

The machine under consideration is subject to random breakdowns. At any failure instant, there are two types of action to be taken: the machine can be repaired or can be replaced by a new identical one. The machine undergoes an imperfect repair that brings it back to the operating condition. The repair is imperfect in that the whole system is not replaced, but only some of its components. However, the machine after repair has the same failure rate as a brand-new one, but it takes longer repair time from one failure to another to obtain that operation condition. The preventive maintenance can be carried out previous to failure and aims at slowing down the deterioration rate, reducing breakdowns risk and repair times durations. We shall call mode of the machine any of the four situations: in operation, in repair, in replacement and in preventive maintenance. These modes are denoted by $1,2,3$ and 4 , respectively. 
The machine's mode changes from 'operation' to 'preventive maintenance' with a transition rate denoted by $q_{14}()=.\omega($.$) . The rate q_{14}($.$) is assumed to be a control variable. The$ inverse of the control variable q14 represents the expected delay between a call for the technician and his arrival. Thus, the preventive maintenance of the machine is triggered after a random delay. During that lead time, there is a request for a maintenance specialist, which includes information to enable the specialist to take with him the tools required for eliminating a specified malfunction or undesired condition. This modeling of the preventive maintenance has been used by authors amount which Boukas and Haurie (1990), Kenne and Nkeungoue (2008). In many other studies in literature, the preventive repair or replacement of a component is modeled as an impulsive control which triggers an immediate jump of the controlled process. The inclusion of a random delay between the call for a technician and his arrival time does not seem more unrealistic than an immediate response. The machine's mode changes from 'preventive maintenance' to 'operation' with a constant transition rate of $q_{41}$. Repair and replacement are two kinds of failure mode and are performed after a failure. The failure mode is denoted by $F$. Therefore, the machine's mode changes from 'operation' to 'failure' at rate $q_{1 F}(a(t))$, which is an increasing function of the machine's age $a(t)$. If the machine is to be repaired, then the transition rate from 'operation' to 'repair' $q_{12}(a(t))$ will be equal to the failure rate $q_{1 F}(a(t))$. Otherwise, the machine will be replaced. The corresponding transition rate from 'up' to 'replacement', $q_{13}(a(t))$, will be equal to the failure rate $q_{1 F}(a(t))$. The machine's mode changes from 'repair' to 'operation' with a transition rate of $q_{21}(n)$, which is a decreasing function of the number of failures $n$. The transition rate from 'replacement' to 'operation' is described by $q_{31}$. All other transition rates equal zero. The state-space diagram of this semi-Markov process is illustrated in Fig 1.

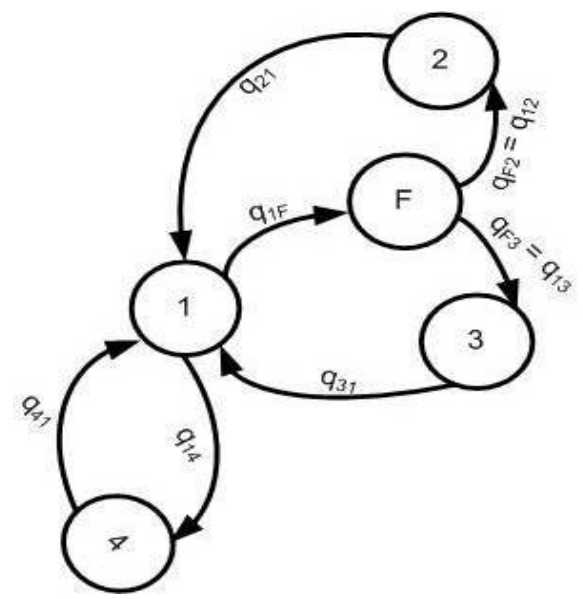

Fig. 1: Machine state-space diagram

It follows that

$$
\left\{\begin{array}{l}
q_{12}(a(t))+q_{13}(a(t))=q_{1 F}(a(t)) \\
q_{12}(a(t))=\left(1-\text { Ind }\left\{q_{13}(a(t))>0\right\}\right) * q_{1 F}(a(t))
\end{array}\right.
$$

where 


$$
\operatorname{Ind}(f(.))= \begin{cases}1 & \text { if the } f(.) \text { is realized } \\ 0 & \text { otherwise }\end{cases}
$$

for any function $f($.). Equation (1) states that, when a failure occurs, the machine cannot be sent to repair and slated for replacement at the same time.

Let $T_{21}(n)=\operatorname{MTTR}(n)$ be the mean repair time after the $n^{\text {th }}$ failure and let us call $q_{21}^{m}(n)$ the repair rate and $\operatorname{MTTR}^{m}(n)$ the mean repair time if preventive maintenance actions are triggered before the $n^{\text {th }}$ failure. We assume that the machine has experienced its $(n-1)^{t h}$ failure and has been repaired. As demonstrated in the literature, a monotone process such as an arithmetic-geometric approach is considered to be relevant, realistic and appropriate to the modelling of a deteriorating system maintenance problem, i.e., for modelling the survival time after the $(n-1)^{\text {th }}$ repair and the repair time after the $n^{\text {th }}$ failure. For more details, we refer the reader to the work of Leung (2006), Zhang et al. (2001) and Badia and Berrade (2009), where some examples of arithmetic-geometric processes are provided.

\subsection{Industrial context}

The study presented in this paper has many applications, especially in production industry. As state by Badia and Berrade (2009), many engineering systems are subject to the so-called unrevealed failures. The unrevealed failures are constituted of those that are detected only by special tests, inspection or monitoring. Seal machines, filling machines, machining centers, grinders, milling, and many of tools machines are among examples. They have a large number of components (treadmill, ball screws, spindle heads, precision gear boxes, axis drive components, rotary tables, saddles, pallets and short). Those components stochastically deteriorate over time and hence the machine. Very often, parts of such machines are broken or damaged, although the machine is operational. For example, worn nozzle, abrasives in pumped liquid, relief valve stuck, partially plugged or improperly adjusted, cavitations, worn bearing do not necessarily stop a liquid filling machine. A worn bearing will for example create knocking noise, while the machine still functioning. Thus, the failure of a machine is due to one or more removable and reparable components. Over time, the number of components to check and repair at failure increases and thus the mean repair time. A preventive maintenance could serve to clean and adjust valves, control stroke and flow injection, replace worn nozzle with properly sized nozzle. By doing that, fewer components are to be checked and repaired at the next failure and consequently the mean repair time is reduced. Preventive interventions intend to reduce breakdowns risk and to maintain the smooth functioning of the machine. Moreover, worn but still functional components are replaced so that when the next failure occurs, the number of components to check, repair or replace is reduced. Therefore, post-repair preventive maintenance durations are reduced.

\section{Repair time reduction and illustration of reduction}

When operating electromechanical equipment, Patton (1995) advised to invest at least half of the corrective maintenance budget in preventive maintenance. Doing this will stop the large number of failures and redirect attention toward doing the job right once. Moreover, detection of failure patterns helps to stop failure modes before they spread to all pieces of equipment. When cracks are detected, they should be eliminated. Thus preventive 
maintenance before failure increases ease of repair. We describe repair times reduction in subsection 3.1 and illustrate examples of reduction in subsection 3.2.

\subsection{Repair times reduction by preventive maintenance}

Let $\operatorname{MTTR}^{m}(n)$ be the post-preventive maintenance mean repair time after the $n^{\text {th }}$ failure. We consider that the effect of preventive maintenance is to reduce the next repair time such that, if there is some preventive maintenance before the $n^{\text {th }}$ failure, the mean repair time at the $n^{\text {th }}$ failure will be of the form $\operatorname{MTTR}^{m}(n)=\phi(\operatorname{MTTR}(n))$, with $0<\phi(\operatorname{MTTR}(n)) \leq \operatorname{MTTR}(n)$.

If the determination of the mean repair time depends on the complete history, we then lose the Markovian property of the system. Hence, $\operatorname{MTTR}^{m}(n)$ depends only on the mean repair time at the $n^{\text {th }}$ failure.

From a practical point of view, when the machine is sent for preventive maintenance, nonfailed but used subsystems are repaired. Hence, at the next failure, fewer subsystems are evaluated and repaired compared to the situation where no preventive maintenance had been performed before failure. Estimation of the repair time reduction factor can be based on preventive maintenance and the repair time's history data or on industrial expert judgment estimation where applicable.

In addition, there are numerous studies of age reduction factor and hazard rate increase factor methods in the literature (Love et al., 2000; Doyen and Gaudoin, 2004; Hariga et al., 2006). Considering that the effect of those methods can result in reduced repair times, one could consider one of the following three reduction models Redf1, Redf2 and Redf3:

Redf1 $\operatorname{MTTR}^{m}(n)=\operatorname{MTTR}(n)-\varepsilon_{0}$ where $\varepsilon_{0}$ is a given constant

Redf2 $\operatorname{MTTR}^{m}(n)=\varepsilon_{n} \operatorname{MTTR}(n)$ with $0<\varepsilon_{n} \leq 1$ after the $n^{\text {th }}$ preventive maintenance

Redf3 $\operatorname{MTTR}^{m}(n)=\varepsilon_{g(n)} \operatorname{MTTR}(n)$ with $\varepsilon_{g(n)}=\frac{g(n)}{g(n)+\eta}$ and $0<\eta<1$ after the $n^{\text {th }}$ preventive maintenance. $g($.$) is a given function (see (Hariga et al., 2006) for more$ details).

The measurement of reduction factors can be obtained by the maximum likelihood method based on historical operating data. We used above models for illustrative purpose. Although all the three models suggested in this paper are analytical, in reality since we are usually talking about hundreds of components and subsystems with very complex interactions and interdependencies, the reduction factors would be very dependent on what kind of preventive maintenance is conducted and what repair time is being affected by that preventive maintenance. For a specific manufacturing system, the typical reduction factors could be determined from post-preventive maintenance activities and repair time's historical data. If a look up table based on engineering design and operator experience is available, it could also serve to measure the post-preventive maintenance effect on repair time. 


\section{2. llustrative reduction factor example}

Let us consider a manufacturing system in which the repair rate following an $n^{\text {th }}$ failure is given by:

$$
q_{21}(n)=q_{0}+q_{1}\left(1-\left(\frac{n-1}{N}\right)^{r}\right) \text { where } q_{1}, q_{0} \text { and } r \text { are given constants. The values used in }
$$

this example are presented in section 5. For a given manufacturing system, they can be determined from the repair time's history data.

The above-mentioned models Redf1, Redf2 and Redf3 will have the effect of improving the availability of the equipment. For the purpose of illustration, we consider that repair times after the preventive maintenance is performed follow the reduction model Redf 2 . Let $\varepsilon_{n}=\varepsilon$ for all $n$.

The effect of Redf2 is to reduce the next repair time with a reduction factor $\varepsilon$ such that, if preventive maintenance is performed before the $n^{\text {th }}$ failure then, the mean repair time at the $n^{\text {th }}$ failure will be $\operatorname{MTTR}^{m}(n)=\varepsilon * \operatorname{MTTR}(n)$, with $\left.\left.\varepsilon \in\right] 0,1\right]$. Thus, there is a one-to-one correspondence between repair time without preventive maintenance (or no reduction) and the post-preventive maintenance repair time.

Figure 2 presents sample paths of the mean repair time for an increasing number of failures. The repair time is a function of the number of failures $n$. Each additional failure requires a longer repair time. Sample paths for different values of $\varepsilon$ are shown in Fig. 2.

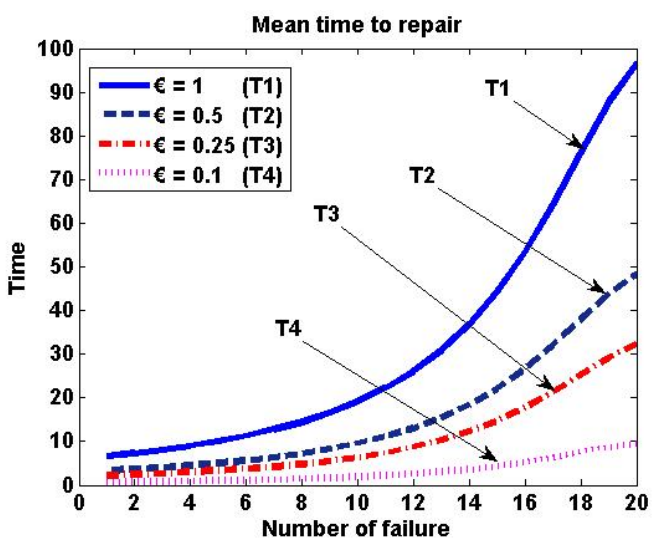

Fig. 2 Mean repair time after preventive maintenance

Figure 2 presents the path of the mean repair time for an increasing number of failures if preventive maintenance is performed before failures, for four $\varepsilon$ values. Note that $T_{1}$ is also the post-preventive maintenance repairs time, with $\varepsilon=1$. This corresponds to the case where there is preventive maintenance before failure, but the mean repair time at failure remains unchanged. This situation arises when preventive maintenance serves to reduce the probability of failures, with no effect on the severity of failures. $T_{2}, T_{3}$ and $T_{4}$ are the paths for $\varepsilon=0.5, \varepsilon=0.25$ and $\varepsilon=0.1$ respectively, and show that the repair time is divided by 2 , 4 and 10 , respectively. 
We can observe from Fig. 2 that the effect of the reduction factor on repair times is more noticeable when the machine experiences a greater number of failures. For the first few failures, curves $T_{2}, T_{3}$ and $T_{4}$ are almost the same. But for a large number of failures (18, for example), they are very distinctive. This can make a significant difference on the impact of performing preventive maintenance on a manufacturing system's availability and/or on overall costs.

Since we know that the other forms of reduction have the same effect of increasing equipment availability, in the remainder of this work, we will consider repair time reduction factor Redf2. We will also consider $\varepsilon_{n}=\varepsilon$ for all $n$, and call the corresponding model arithmetic repair time reduction.

\section{Optimal control problem and optimality conditions}

The following subsections present the optimal control problem and optimality conditions.

\subsection{Optimal control problem}

The state of the system is described by the machine mode $\xi(t) \in \Omega=\{1,2,3,4\}$ with the transitions shown in Fig. 1, the number of failures $n(t)$ and the age of the machine $a(t)$.

The age of the machine $a(t)$ is an increasing function of chronological time and is described by the following differential equation:

$$
\frac{d a(t)}{d t}=\delta ; a(T)=0
$$

where $0<\delta \leq 1$ is a given constant and $T$ the time of the last restart of the machine. If $\delta=1$, then the age of the machine is the cumulative working time since the last restart of the machine.

Recall that the failure rate $q_{1 F}($.$) is an increasing function of a machine's age a(t)$. It is reset to its initial value after a repair or preventive maintenance, but that does not mean that the machine is new.

We assume that the following constraint holds for the preventive maintenance transition rate:

$$
0 \leq \omega(.) \leq \omega_{\max }
$$

where $\omega_{\max }$ is the maximum rate of preventive maintenance. The inverse of $\omega($.) represents the expected delay between the decision to perform preventive maintenance actions and the effective switch from operation mode to preventive maintenance mode.

We define $q_{12}^{S}(a)=q_{12}(a) \times$ Ind $\{a \leq s\}$ and $q_{13}^{S}(a)=q_{13}(a) \times \operatorname{Ind}\{a>s\}$ if, after a failure occurs, the machine is replaced after age $s$. The parameter $s$ is the repair/replacement switching threshold age. That is, on the $n^{\text {th }}$ failure, if the age of the machine is above the threshold value $s$, the machine is replaced; if it is not, corrective maintenance is conducted. 
We shall refer to $\{\xi(t): t \geq 0\}$ as the stochastic process with value in $\Omega$ and $Q^{S}(\cdot)=\left(q_{\alpha \beta}^{S}().\right)$ as the $4 \times 4$ matrix such that $q_{\alpha \beta}^{S}(a) \geq 0$ if $\alpha \neq \beta, q_{11}^{S}()=.q_{11}($.$) ;$ $q_{31}^{S}()=.q_{31}(.) ; q_{14}^{S}()=.q_{14}(.) ; q_{41}^{S}()=.q_{41}($.$) ; and q_{\alpha \alpha}^{S}()=.-\sum_{\alpha \neq \beta} q_{\alpha \beta}^{S}(.) . \quad Q^{S}(\cdot)$ is the transition matrix of the semi-Markov chain $\xi(\cdot)$

Let us define $G(\alpha, a, n)$ as the running cost of being in state $\alpha$, at age $a$ of a machine that has already had its $n^{\text {th }}$ failure.

The expected discounted cost is given by:

$$
J(\alpha, a, n, s, \omega)=E\left[\int_{0}^{\infty} e^{-\rho t} G(\cdot) d t / \xi(0)=\alpha, a(0)=a, n(0)=n\right]
$$

where $\rho$ is the discounted rate used to make the costs incurred at future dates less important than the cost incurred today.

Let $\Gamma=\left\{\pi=(s(),. \omega()):. 0 \leq s() \leq\right.$.$\left.M and 0 \leq \omega(.) \leq \omega_{\max }\right\}$. Any plan $\pi=(s, \omega) \in \Gamma$ is called an admissible plan, and our problem is to minimize the expected discounted cost, given by equation (4), for any admissible plan.

The control variables are the threshold age $s$ after which the machine should automatically be replaced at the next failure and the preventive maintenance rate $\omega$.

Optimal policies are obtained by searching:

$$
V(\alpha, a, n)=\min _{(s, \omega(\cdot)) \in \Gamma} J(\alpha, a, n, \omega, s)
$$

We define $S_{n}$ as the optimal replacement policy before the $n^{\text {th }}$ failure. That is, for each $n$, there exists an age $S_{n}$ such that if $a(t) \geq S_{n}$, the optimal action is to replace the machine at the $n^{\text {th }}$ failure.

Let $N_{m}$ be the minimum number of failures that occur before a systematic replacement at the next failure, regardless the machine's age. Note that when $n=N_{m}$, then $S_{n}=0$; it is therefore sufficient to determine $S_{n}$ instead of both variables.

Our objective is then to determine the age $s_{n}$ before systematic replacement at the next failure and the preventive maintenance control $\omega(\cdot)$ that will minimize $J(\cdot)$.

As established in the literature (Makis and Jardine, 1993; Love et al., 2000), we can choose an upper bound on the age, $M$ to be very large compared to $S_{1}$, beyond which the system is automatically replaced. 
The value function $V(\alpha, a, n)$ satisfies specific properties called optimality conditions, which are presented in the next section.

\subsection{Optimality conditions}

The value function $V(\alpha, a, n)$ satisfies a set of coupled partial derivative equations derived from the application of the dynamic programming approach called the Hamilton-JacobiBellman (HJB) equations given by equations. (6):

$$
\rho V(\alpha, a, n)=\underset{(s, \omega) \in \Gamma}{\operatorname{Min}}\left\{G(.)+\frac{\partial}{\partial a} V(\alpha, a, n) \operatorname{Ind}\{\xi(t)=1\} \frac{d a(t)}{d t}+\sum_{\beta \neq \alpha} q_{\alpha \beta}^{S}(a, n)[V(\beta, \varphi(a, ; n), \psi(n))-V(\alpha, a, n)]\right\}
$$

where $\alpha \in \Omega$;

$\varphi(a, \xi, n)=\left\{\begin{array}{cc}0 & \text { if } \xi\left(\tau^{+}\right)=1 \text { and } \xi\left(\tau^{-}\right)=2,3 \text { or } 4 \\ a\left(\tau^{-}\right) & \text {otherwise }\end{array}\right.$ and

$\psi(n)=\left\{\begin{array}{ll}0 & \text { if }\left\{\xi\left(\tau^{+}\right)=1 \text { and } \xi\left(\tau^{-}\right)=3\right\} \\ n+1 & \text { if }\left\{\xi\left(\tau^{+}\right)=2,3 \text { and } \xi\left(\tau^{-}\right)=1\right\} \\ & \text { or }\left\{\xi\left(\tau^{+}\right)=1 \text { and } \xi\left(\tau^{-}\right)=2\right\} \\ n & \text { otherwise }\end{array} ; \frac{\partial}{\partial a} V(\alpha, a, n)\right.$ is the gradient of the value function $V(\alpha, a, n)$.

The HJB equations (6) are solve by approximating $V(\alpha, a, n)$ with a function $V^{h_{a}}(\alpha, a, n)$ and the first-order partial derivatives of the value function $\frac{\partial}{\partial a} V(\alpha, a, n)$ by: $\frac{\partial}{\partial a} V(\alpha, a, n)=\frac{1}{h_{a}}\left[V^{h_{a}}\left(\alpha, a+h_{a}, n\right)-V^{h_{a}}(\alpha, a, n)\right]$

where $h_{a}$ is a discrete increment associated with state variable $a$.

Let us define $Q_{h_{a}}^{\alpha}=\left|q_{\alpha \alpha}^{S}\right| ; \quad P_{a}(\alpha)= \begin{cases}\frac{\sigma}{h_{a} Q_{h_{a}}^{\alpha}} & \text { if } \alpha=1 \quad \text { and }{ }^{\beta}(\alpha)=\frac{{ }^{q_{\alpha \beta}}}{Q_{h_{a}}^{\alpha}} \\ 0 & \text { otherwise }\end{cases}$

The HJB equations can be rewritten as follows:

$$
V^{h_{a}}(\alpha, a, n)=\operatorname{Min}_{(s, \omega) \in \Gamma}\left\{\frac{G(.)}{Q_{h_{a}}^{\alpha}\left(1+\frac{\rho}{Q_{h_{a}}^{\alpha}}\right)}+\frac{1}{\left(1+\frac{\rho}{Q_{h_{a}}^{\alpha}}\right)}\left(P_{a}(\alpha) V^{h_{a}}\left(\alpha, a+h_{a}, n\right)+\sum_{\beta \neq \alpha} P^{\beta}(\alpha) V^{h_{a}}(\alpha, a, n)\right\}\right.
$$

The step $h_{a}$ is chosen such that

$$
\lim _{h_{a \rightarrow 0}} V^{h_{a}}(\alpha, a, n)=V(\alpha, a, n)
$$

We use the policy improvement technique to obtain a solution of the approximating optimization problem. We refer the reader to (Kushner and Dupuis, 1992) for the algorithm of this technique. In the next section, we illustrate the approach developed in this paper, using a numerical example. 


\section{Numerical example}

We consider the computational domain $D$ defined by: $D=\{(a, n): 0 \leq a \leq 100 ; 0 \leq n \leq 20\}$. The costs of preventive maintenance $\left(C_{m}=100\right)$ and replacement $\left(C_{\text {rem }}=20000\right)$ are assumed to be constant. Costs are in dollars.

Let $C_{r e p}$ be the cost of repair per unit of mean time. Thus the mean repair cost is $C_{r e p} * T_{21}(n)$. Recall that $T_{21}(n)$ is the mean repair time before the $n^{\text {th }}$ failure. Hence, the cost function $\mathrm{G}($.$) is given by:$

$G(\alpha, a, n)=C_{r e p} * T_{21}(n) * \operatorname{Ind}\{\xi(t)=3\}+C_{r e m} * \operatorname{Ind}\{\xi(t)=2\}+C_{m} * \operatorname{Ind}\{\xi(t)=4\}$.

Given the frequent utilization of the Weibull distribution in reliability engineering (Love et al., 2000), we assume that the lifetime of a new machine follows a Weibull distribution. The parameter scale is $\lambda=0.03$ and the shape parameter $\alpha=2$.

Table 1 summarizes other parameters used in this paper.

\begin{tabular}{|l|c|c|c|c|c|c|}
\hline Parameter & $N$ & $q_{0}$ & $q_{1}$ & $q_{31}$ & $\mathrm{r}$ & $\delta$ \\
\hline Value & 20 & 0.01 & 0.14 & 10 & 2 & 1 \\
\hline Parameter & $c_{\text {rep }}$ & $\mathrm{h}_{\mathrm{a}}$ & $\rho$ & $q_{41}$ & $\omega_{\max }$ & $\varepsilon$ \\
\hline Value & 100 & 0.2 & 0.05 & 0.2 & 0.1 & 0.1 \\
\hline
\end{tabular}

Table 1. Parameters of the numerical example

For $\delta=1$, as in Table 1, the age of the machine is the chronological working time since the last restart of the machine. Recall that the lifetime distribution of a new machine follows a Weibull distribution with the density function $f(a)=\lambda^{\alpha} \alpha a^{\alpha-1} \exp \left(-(\lambda a)^{\alpha}\right)$. Its cumulative distribution function is $F(a)=1-\exp \left(-(\lambda a)^{\alpha}\right)$.

At any time, we consider that the system has experienced its $(n-1)^{\text {th }}$ failure, $n=1,2,3, \ldots$ and has been repaired. Since the repair restores the age of the machine to zero (but the machine is not new), the mean time to failure (MTTF) of the machine before the $n^{\text {th }}$ failure is equal to the mean time to first failure and is given by $\operatorname{MTTF}=(1 / \lambda) \Gamma\left(1+\frac{1}{\alpha}\right)$, where $\Gamma($.$) is the gamma function.$

Using the previous parameters, $M T T F=29.5$ units of time. Thus, our selection of the upper bound for the control limits on age is $M=100$. The probability that the machine will have its first failure before $M=100$ is almost equal to 1 , as illustrated by the graph of the distribution function in Fig. 3. 


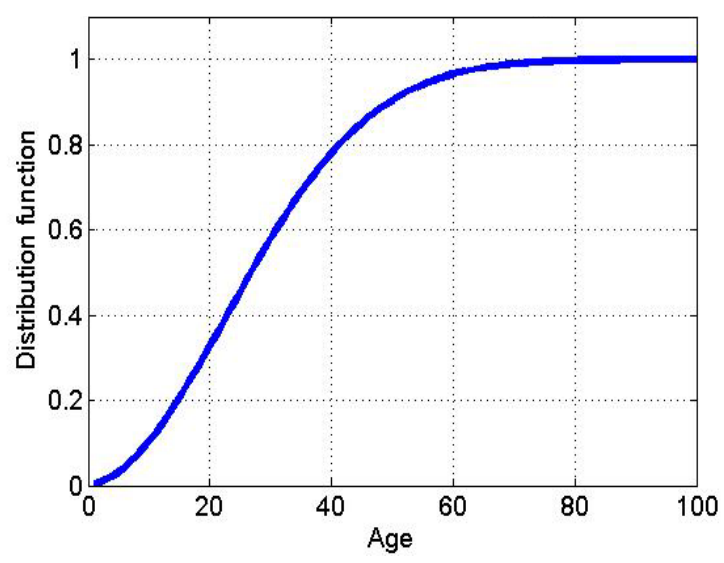

Fig. 3 Distribution function of failures over time for a given failure number $n$

This ensures that if the replacement threshold age at first failure of the machine is greater than $M=100$, that machine will never be replaced after a first failure. In these circumstances, it is not necessary to have $M>>S_{1}$, as is the case in (Love et al., 2000), because $M$ and $S_{1}$ appear to be infinity for the machine.

For $\varepsilon=0.1$, there is a strong incentive to perform preventive maintenance because it will reduce the next repair time by a factor of 10 . Moreover, if no preventive maintenance is carried out before a large number of failures, the mean time to repair for each failure will follow the path P1 of Fig. 4 below. When preventive maintenance is triggered once before each failure, then the path of the mean repair time follows $\mathrm{P} 2$.

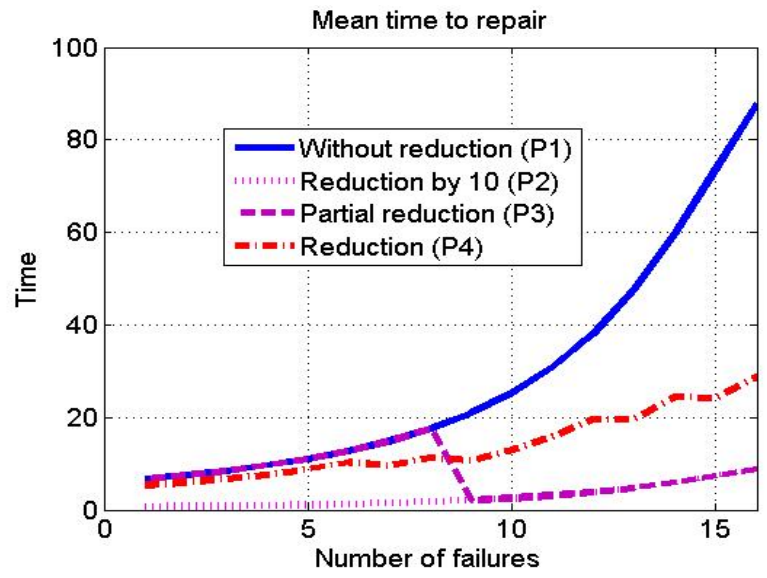

Fig. 4 Mean repair time and its possible reduction

Note that in reality, preventive maintenance may not be performed before every failure. Thus, the real path $\mathrm{P} 3$ of the mean repair time could be composed of $\mathrm{P} 1$ and $\mathrm{P} 2$, as presented in Fig. 4. For relatively small reduction factors, preventive maintenance may be done more than once between failures. The corresponding reduced repair time follows P4.

The policy improvement technique is used to solve equation (7). The results obtained for the 
values given in Table 1 are presented in Figs. 5 to 7.

The repair/replacement policy $S_{n}$ presented in Fig.5 divides the plan $(a(), n$.$) into two zones:$ Zone 1 below $S_{n}$ and Zone 2 above $S_{n}$.

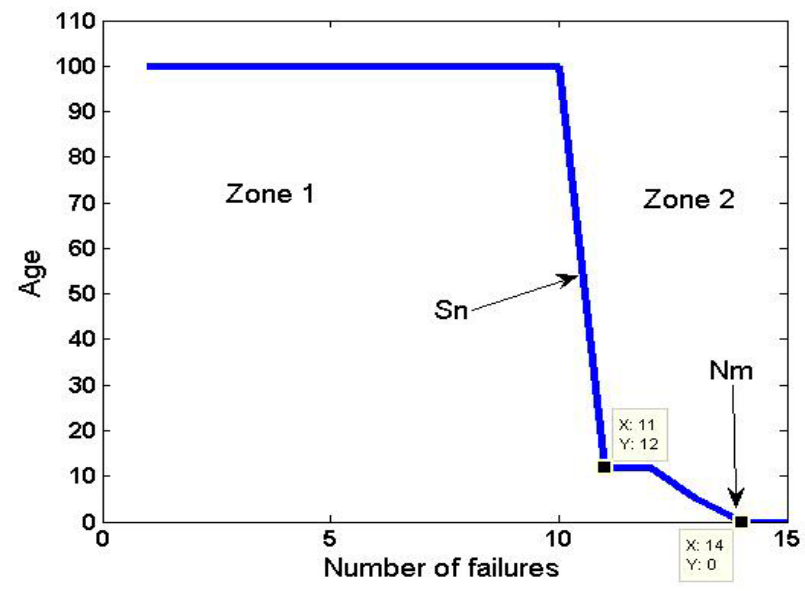

Fig. 5 Repair/replacement policy

If a failure occurs when the system is located in Zone 1 of Fig.5, the optimal action is to repair the machine. Otherwise, the optimal action is to replace the machine. Since the decisions to repair or to replace the machine are limited to instances of machine failure, Fig. 5 indentifies the action to be undertaken if the location of the system at failure is known.

The machine would have at least $N_{m}=14$ failures before systematic replacement, regardless of its age, will occur at the next failure. Recall that the age $a=100$ appears to be infinity for the machine. We can conclude from Fig. 5 that the machine will never be replaced before it has experienced at least 10 failures.

Let $R_{n}(a(t))$ denote a function with a value of 1 if a repair action is undertaken after the $n^{\text {th }}$ failure occurs at age $a(t)$, and 0 if it does not. The above results enable us to illustrate the repair/replacement policy as: upon the $n^{\text {th }}$ failure of the machine at age $a(t)$,

$$
R_{n}(.)= \begin{cases}1 & \text { if } a(.) \leq S_{n} \\ 0 & \text { otherwise }\end{cases}
$$

with $S_{n}$ given in Fig. 5 .

The preventive maintenance policy is presented in Fig. 6 below. 


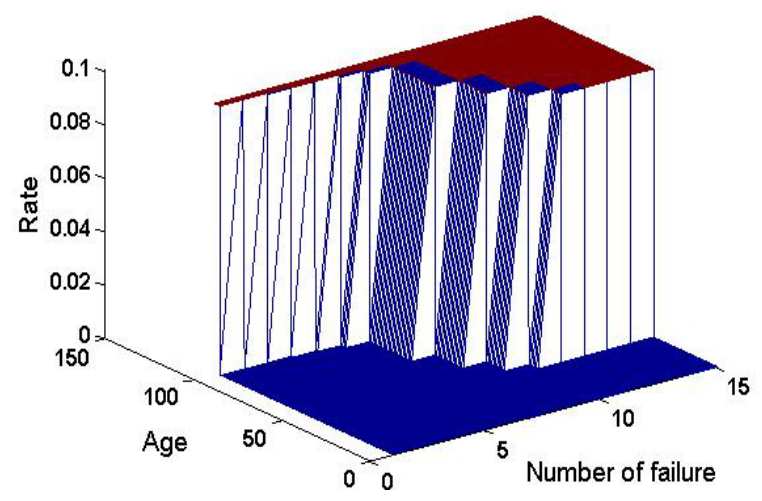

Fig. 6 Preventive maintenance policy

The preventive maintenance policy given by Fig. 6 will be represented in the remainder of the paper by its boundary, as shown in Fig. 7 .

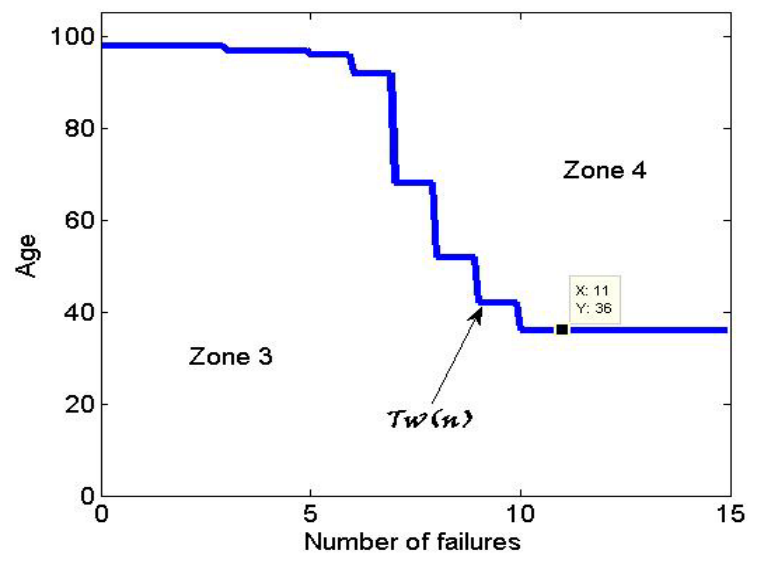

Fig. 7 Boundary of the preventive maintenance policy

Figure 7 identifies two zones delimited by the boundary $T_{\omega}(n)$. The preventive maintenance actions at each failure number are triggered according to the age limit policy described in Fig. 7. Decisions to send the machine to preventive maintenance are undertaken when the machine is operational. If the machine's state is located in Zone 3, that is, the age and number of failures are below the boundary $T_{\omega}(n)$, there is no need to call a maintenance specialist. Conversely, if the machine's state is in Zone 4, which is above the boundary $T_{\omega}(n)$, the optimal response is to trigger preventive maintenance actions. When there is a decision to perform a preventive maintenance, if for example $\mathrm{w}()=$.0.3 , the machine is shutdown for preventive maintenance after $1 / 0.3$ units of times, corresponding to the delay between the call of a technician and its arrival. If there is no preventive maintenance scheduled at a given time, $\mathrm{w}()=$.0 and the machine continue to produce. The proposed preventive maintenance policy is a feedback control policy based on machine's age and number of failures.

According to the results provided by Fig. 7, the trend is to send old machines (determined by age and the number of breakdowns that have occurred) to preventive maintenance, which is the reality in manufacturing systems.

The preventive maintenance actions are triggered according to the age limit policy described in Fig. 7, which indicates that preventive maintenance should be performed at rate $\omega($.$) ,$ 
with

$$
\omega(.)= \begin{cases}0 & \text { if } a(.) \leq T_{\omega}(.) \\ \omega_{\max } & \text { otherwise }\end{cases}
$$

where $T_{\omega}($.$) is the age limit for preventive maintenance before the next machine failure. For$ a given failure number $n, T_{\omega}(n)$ is provided by Fig.7.

It can be observed that when the number of failures increases, the age limit $T_{\omega_{n}}=T_{\omega}(n)$ decreases, meaning that the machine is sent to preventive maintenance more often.

On the basis of the previous results, we can illustrate the repair/replacement and preventive maintenance policies by the vector $\left(S_{n}, N_{m}, T_{\omega_{n}}\right)$, where $T_{\omega_{n}}$ corresponds to the value of $T_{\omega}($.$) for each n$ value in Fig. 7 , and $N_{m}=\min \left\{n \geq 0\right.$ such that $\left.S_{n}=0\right\}$.

\section{Model implementation}

For the implementation of the obtained results, Fig.5 and Fig.7 are used. At initial time, a new machine is installed. Its age is 0 and the number of failure already occurred is 0 . As soon as the machine is put into use, its age starts increasing. While waiting the first failure of the machine, if its age reaches $T_{\omega_{1}}$ (in this example $T_{\omega_{1}}=100$ ) ), send the machine to preventive maintenance. When the first failure occurs, if the age of the machine is below $S_{1}$ (here $S_{1}=100$ ), repair the machine; otherwise, replace the machine by a new identical one. Recall that the age 100 appears to be infinite for the machine, it is certain that the first failure will occur below 100. That means that the machine won't be sent to preventive maintenance before the first failure nor be replaced after the first failure. Thus, after the first failure, the machine is repaired and no repair time reduction is considered.

Now, suppose that the machine has already had its $10^{\text {th }}$ failure and has been repaired. Upon the restart of the machine, and while waiting the $11^{\text {th }}$ failure, actions to be taken are illustrated in Fig. 13.

While waiting the $11^{\text {th }}$ failure, actions to be taken are function of the age of the machine as shown in Fig. 13. When operating the machine, if no failure occurs before age 36, continue operating the machine. When the age of the machine reaches 36 without failure, send the machine to preventive maintenance. The aim of performing preventive maintenance is to reduce the need for corrective maintenance and keep the machine in its operating state. If a failure occurs after preventive maintenance has been done, the mean repair time will systematically be reduced compare to the case where no preventive maintenance has been done. At the $11^{\text {th }}$ failure, if the age of the machine is less than 12, repair the machine, otherwise replace the machine. Replacement restores the machine to its brand-new condition at time zero. Conversely, after repair the machine is not new and will certainly experience a $12^{\text {th }}$ failure. The maximum number of failures the machine could experience is 15 . Thus, the machine is systematically replaced after the $15^{\text {th }}$ failure regardless of its age. Although not specified in the diagram, one should remember that repair time following preventive maintenance is reduced. 


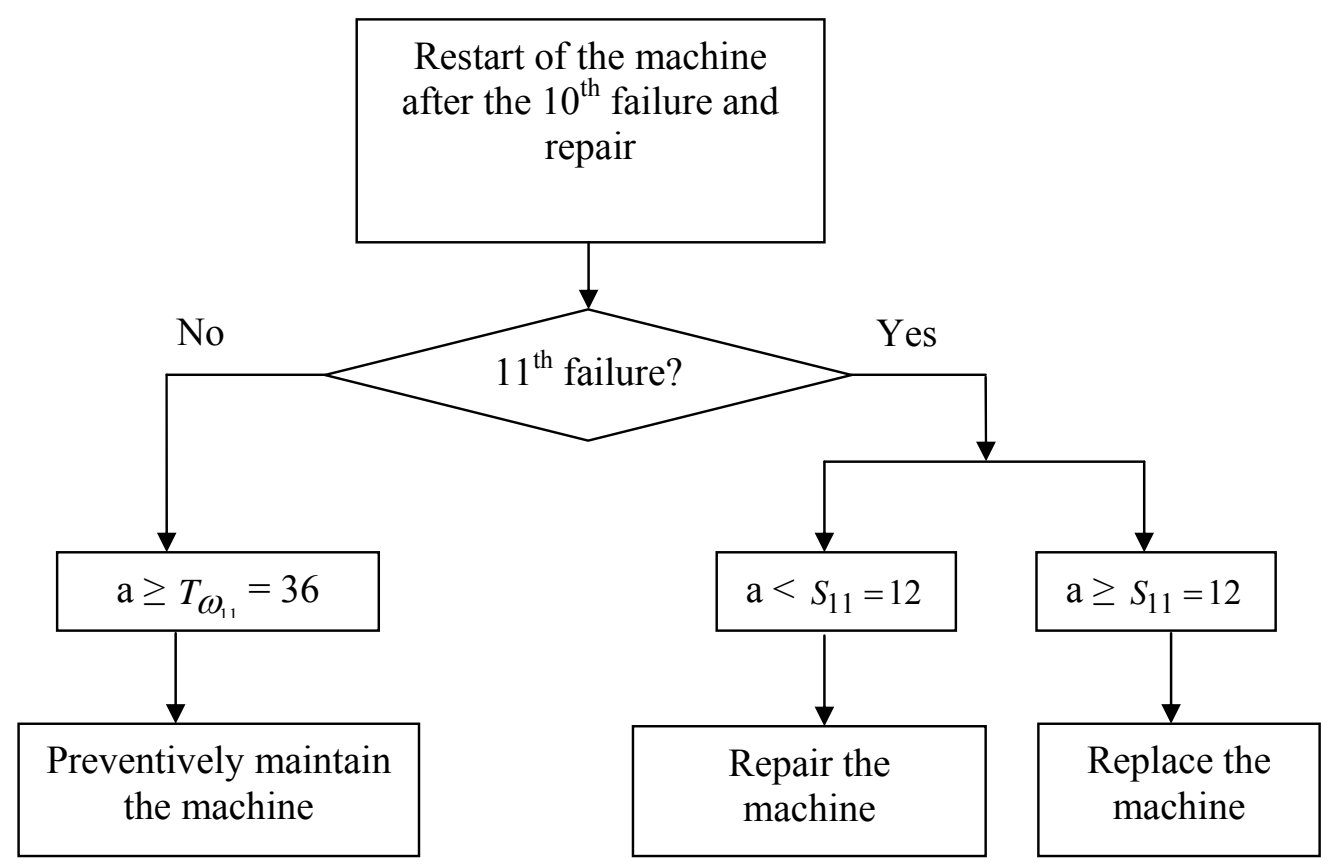

Fig. 13 Model implementation diagram

To see how these results are influenced by some of the parameters used in this paper, in the next section we present a sensitivity analysis performed according to the previous results.

\section{Results and sensitivity analysis}

The previous section provided the repair/replacement and preventive maintenance policies $\left(S_{n}, N_{m}, T_{\omega_{n}}\right)$, which recommend replacing the machine at the $N_{m}{ }^{\text {th }}$ failure or at failure after age $S_{n}$, whichever comes first, and not performing preventive maintenance before age $T_{\omega_{n}}$ is reached. The decisions to repair, to replace or to trigger preventive maintenance are based on the overall incurred cost. We analyze the sensitivity of those policies according to repair cost per unit of time, replacement cost and preventive maintenance cost in the first subsection. In the second subsection, we examine the sensitivity of the optimal policies to the reduction of the repair time when preventive maintenance is carried out.

\subsection{Sensitivity analysis of repair, replacement and preventive maintenance costs}

In this subsection, we will perform sensitivity analysis on the repair cost per unit of time, the replacement cost and the cost of preventive maintenance.

When the repair cost per unit of time takes the four values 50,100,150 and 200, we obtain the results presented in Figs. 8a. and 8b. On the basis of Fig. 8a., we conclude that when repair costs increase, the machine is replaced earlier. According to Fig. 8b., preventive maintenance actions are also triggered earlier. 


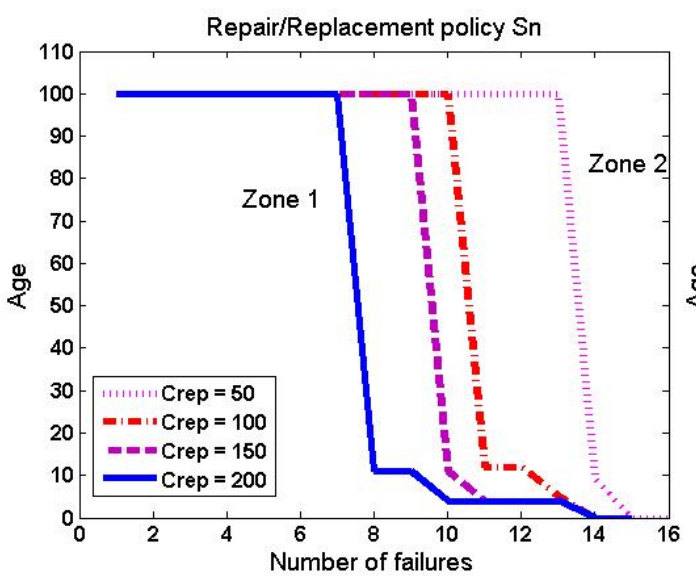

a. $S_{n}$

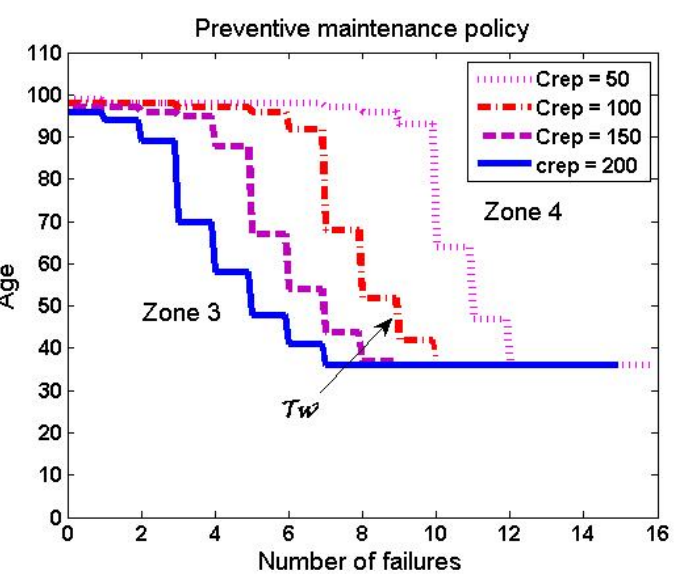

b. $T_{\omega_{n}}$

Fig. 8 Sensitivity of policies to the variation of repair cost per unit of time

The trend is to reduce the repair time by recommending sending a machine to preventive maintenance earlier (Zones 2 and 4 increase).

As we can see from Fig. 9a., Zone 1 increases as the machine replacement costs increase. That is, the higher the replacement cost, the higher the threshold age and the number of failures expected before systematic replacement.

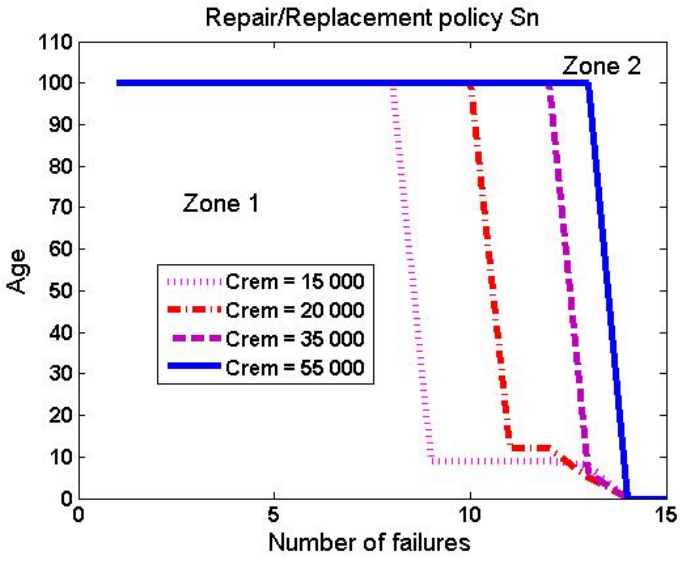

a. $S_{n}$

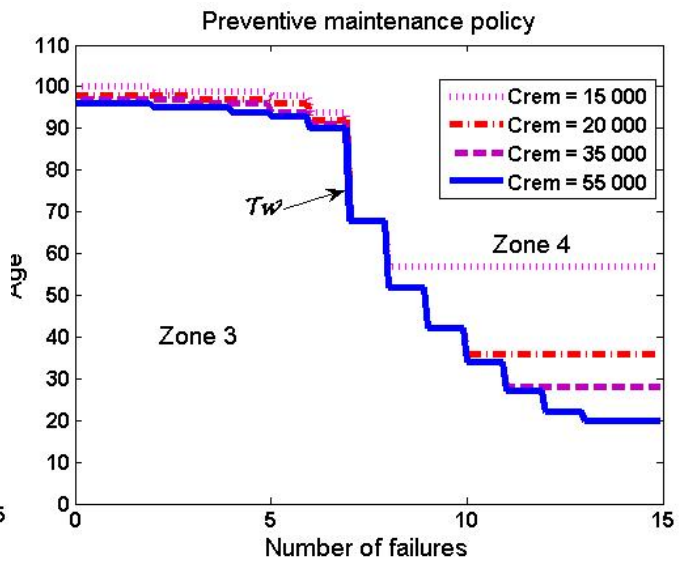

b. $T_{\omega_{n}}$

Fig. 9 Sensitivity of policies to the variation of repair cost per unit of time

The corresponding preventive maintenance policies given by Fig. 9b. show that as replacement cost increases, preventive maintenance is triggered earlier. The size of Zone 4, where preventive maintenance is recommended, increases. However, it can be observed from both Fig. 9a. and Fig. 9b. that increasing the replacement cost over the range [15 000, 55000 ] does not have a significant effect on the policies when the number of failures is less than 9, at least for the numerical examples considered in this study. Increasing replacement costs decrease Zones 2 and 3: replacement is postponed while preventive maintenance is triggered more often.

Since there is an incentive to perform preventive maintenance, we made a variation of 
preventive maintenance costs from low to moderate values as illustrated by the graphs in Figs. 10a. and 10b.

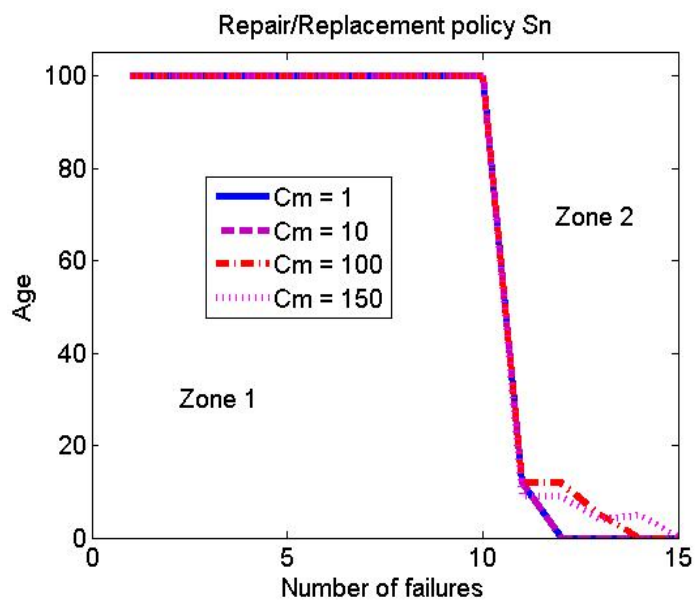

a. $S_{n}$

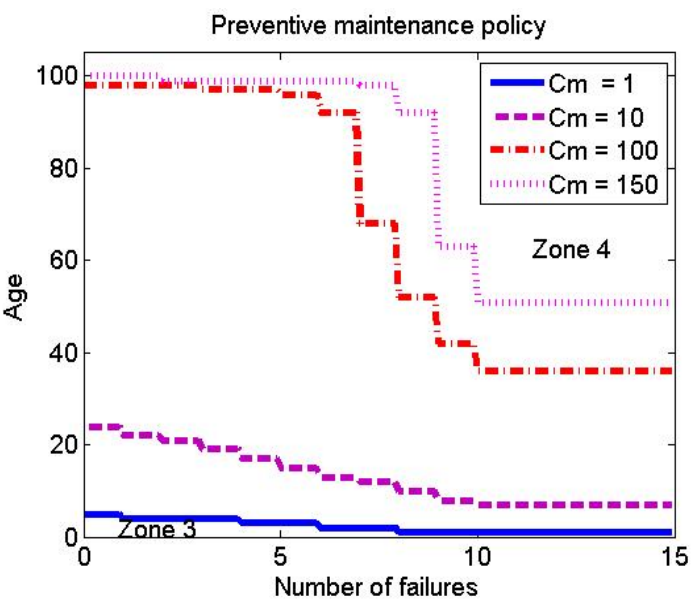

b. $T_{\omega_{n}}$

Fig. 10 Variation of preventive maintenance cost per unit of time

Decreasing preventive maintenance costs keeps Zone 1 virtually the same until the machine experiences its $10^{\text {th }}$ failure. Conversely, it practically eliminates Zone 3. Figure 10a. shows that the repair and replacement policy is less sensitive to preventive maintenance cost variations. As indicated in Fig. 10b., decreasing preventive maintenance cost triggers more frequent preventive maintenance. These results are realistic because preventive maintenance costs are very small compared to replacement costs.

\subsection{Sensitivity analysis of the variation of the reduction factor}

In Fig. 11, the reduction factor ( $\varepsilon$ ) takes four values: $0.1,0.25,0.5,1$. When $\varepsilon=1$, the mean repair time remains unchanged after preventive maintenance. From Fig. 11, the machine experiences only 4 failures before systematic replacement at failure. In this case, $M>>S_{1}$. Recall that the mean time to failure is 29.5 units of time and $S_{1}<29.5$ units of time. Such a machine could be replaced after a first failure if its age is greater than 20 units of time.

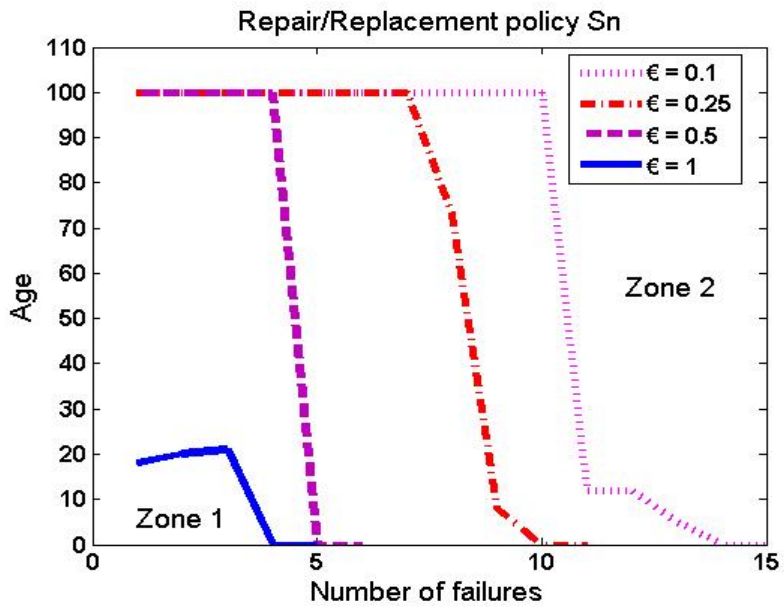

Fig. 11 Repair/replacement policy for several values of the reduction factor 
When $\varepsilon=0.1$, the mean repair time after a failure occurs is divided by 10 if preventive maintenance had been performed before failure. The machine experiences many more failures $\left(N_{m}=14\right)$ before systematic replacement after a failure. On the other hand, with such a system, the machine could never be replaced before it experiences 10 failures. The $\varepsilon$ values between 0.1 and 1 confirm the trend that the smaller the $\varepsilon$, the more the machine experiences failures, and that performing preventive maintenance really increases the lifetime of the machine.

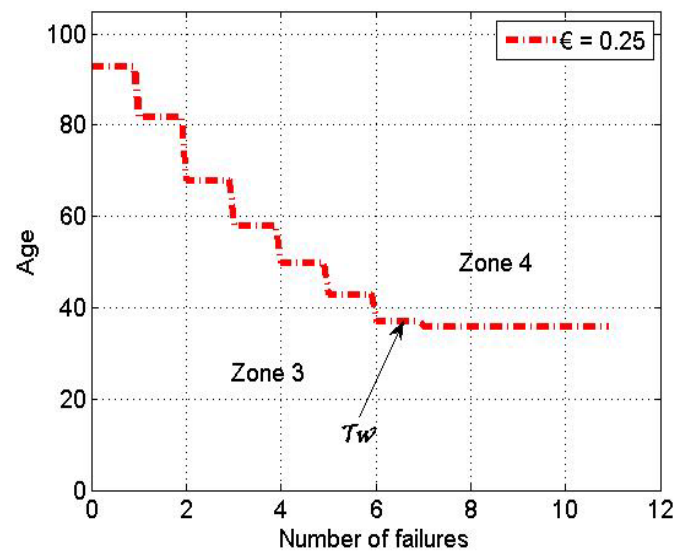

a. $\varepsilon=0.25$

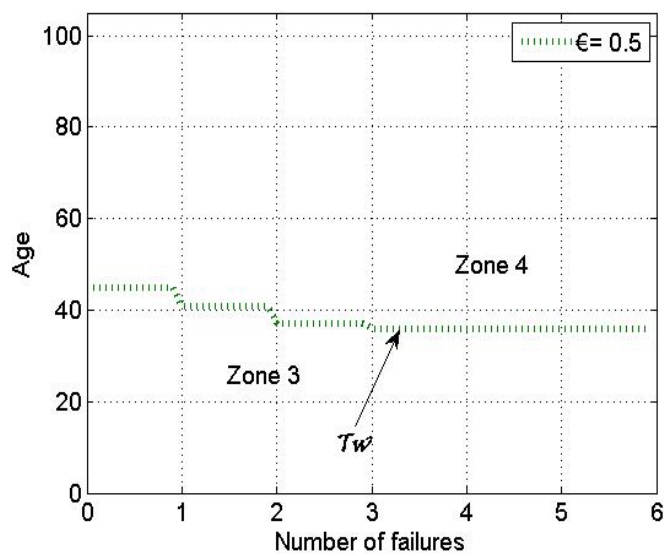

b. $\varepsilon=0.5$

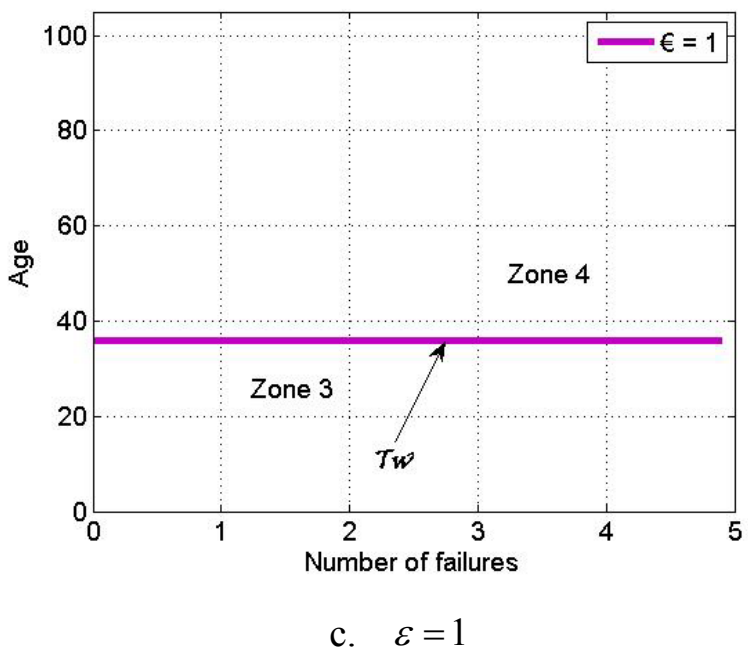

Fig. 12 Preventive maintenance policy for several values of the reduction factor

The preventive maintenance policies for $\varepsilon=0.25 ; 0.5$; and $\varepsilon=1$ are illustrated in Figs. 12 a-c. They show that although the machine is replaced early, preventive maintenance is somewhat recommended if the machine has reached a certain age.

The above sensitivity analysis confirms that the general repair/replacement and preventive maintenance policies $\left(S_{n}, N_{m}, T_{\omega_{n}}\right)$ are threshold-type, and they recommend replacing the machine at the $N_{m}^{\text {th }}$ failure or at failure after age $S_{n}$, whichever comes first, and to not perform preventive maintenance before age $T_{\omega_{n}}$. If at the $n^{\text {th }}$ failure, the age of the 
machine is greater than $S_{n}$ or the failure number is above $N_{m}$, then undertake a repair.

The obtained results indicate that the optimal repair/replacement and preventive maintenance policy for the considered manufacturing system is characterized by the aforementioned three parameters (i.e. $S_{n}, N_{m}, T_{\omega_{n}}$ ). Two of those parameters characterize the repair/replacement switching policy (i.e. $S_{n} ; N_{m}$ ) and one parameter characterizes the preventive maintenance policy (i.e., $T_{\omega_{n}}$ ). The overall control policy is given by equations (9-10) and figures 6-7, and is completely defined by the parameter values $\left(S_{n} ; N_{m}\right)$ for repair/replacement switching and $T_{\omega_{n}}$ for preventive maintenance policy.

\section{Conclusions}

In this paper, we investigate the integration of preventive maintenance with the repair/replacement policy for a machine subject to random breakdowns. In addition, failures intensity and repair times increase with the number of failures. We introduce a reduction factor approach to decrease the repair times if preventive maintenance is performed before failures. Such a stochastic control problem is quite complex due to the machine's failure repair and preventive maintenance history. Modeling the system with a semi-Markov process allowed us to take into account the stochastic failure history, thereby adding a new dimension to the repair/replacement theory. We showed that implementing this type of preventive maintenance increases the lifetime of the machine. We illustrated the proposed approach using a numerical example and perform sensitivity analysis to show that the structure of the obtained policies is maintained when parameters change. The obtained results are particularly useful for industrial systems that experience losses due to increasing repair times.

\section{References}

Ansell, J. I., T. W. Archibald and L. C. Thomas (2004). "The elixir of life: using a maintenance, repair and replacement model based on virtual and operating age in the water industry." IMA Journal of Management Mathematics 15(2): 151-160.

Aven, T. and I. T. Castro (2008). "A minimal repair replacement model with two types of failure and a safety constraint." European Journal of Operational Research 188(2): 506515.

Badia, F. G. and Berrade M. D.(2009). "Optimum Maintenance Policy of a periodically Inspected System under Imperfect Repair." Advances in Operations Research 2009, Article ID 691203, 13 pages

Boukas, E.-K. and A. Haurie (1990). "Manufacturing flow control and preventive maintenance: A stochastic control approach." IEEE Transactions on Automatic Control 35(9): 1024-1031.

Doyen, L. and O. Gaudoin (2004). "Classes of imperfect repair models based on reduction of failure intensity or virtual age." Reliability Engineering and System Safety 84(1): 4556.

Hariga, M. A., M. N. Azaiez and M. B. Daya (2006). "A discounted integrated inspectionmaintenance model for single deteriorating production facility." International 
Transactions in Operational Research 13(4): 353-64.

Kenne, J. P. and A. Gharbi (1999). "Experimental design in production and maintenance control problem of a single machine, single product manufacturing system." International Journal of Production Research 37(3): 621-637.

Kenne, J. P. and L. J. Nkeungoue (2008). "Simultaneous control of production, preventive and corrective maintenance rates of a failure-prone manufacturing system." Applied Numerical Mathematics 58 (2008) 2: 180-194.

Kijima, M. (1989). "Some results for repairable systems with general repair." Journal of Applied Probability 26: 89-102.

Kijima, M., H. Morimura and Y. Suzuki (1988). "Periodical replacement problem without assuming minimal repair." European Journal of Operational Research 37(2): 194-203.

Kushner, H. and P. G. Dupuis (1992). Numerical methods for stochastic control problems in continuous time. Springer-verlag. New York.

Lam, Y. (1988). Geometric processes and replacement problem, Acta Mathematicae Applicatae Sinica 4: 366-377

Leung, K. N. F. (2006). "A note on 'A bivariate optimal replacement policy for a repairable system'." Engineering Optimization 38(5): 621-625.

Love, C. E., Z. G. Zhang, M. A. Zitron and R. Guo (2000). "Discrete semi-Markov decision model to determine the optimal repair/replacement policy under general repairs." European Journal of Operational Research 125(2): 398-409.

Lugtigheid, D., D. Banjevic and A. K. S. Jardine (2008). "System repairs: When to perform and what to do?" Reliability Engineering and System Safety 93(4): 604-615.

Makis, V. and A. K. S. Jardine (1991). "Optimal replacement of a system with imperfect repair." Microelectronics and Reliability 31(2-3): 381-388.

Makis, V. and A. K. S. Jardine (1993). "A note on optimal replacement policy under general repair." European Journal of Operational Research 69(1): 75-82.

Malik, M. A. K. (1979). "Reliable preventive maintenance scheduling." AIIE Transactions 11(3): 221-8.

Nakagawa, T. and M. Kijima (1989). "Replacement policies for a cumulative damage model with minimal repair at failure." IEEE Transactions on Reliability 38(5): 581-584.

Nguyen, D.G., Murthy, D.N.P., 1981. "Optimal preventive maintenance policies for repairable systems." Operations Research 29, 1181-1194.

Patton, Joseph D. (1995). "Preventive maintenance. Research Triangle Park, N.C.: Instrument Society of America, ISBN 1556175337.

Radhoui, M., Rezg, N. and Chelbi, A. (2009). "Integrated model of preventive maintenance, quality control and buffer sizing for unreliable and imperfect production systems." Cutting Edge Production Research of the French Community International Journal of Production Research 47(2): 389-402.

Stanley, A. D. J. (1993). "On geometric processes and repair replacement problems." Microelectronics Reliability 33(4): 489-491.

Wang, G. J. and Y. L. Zhang (2006). "Optimal periodic preventive repair and replacement policy assuming geometric process repair." IEEE Transactions on Reliability 55(1): 118- 
122.

Wang, H. (2002). "A survey of maintenance policies of deteriorating systems." European Journal of Operational Research 139(3): 469-489.

Wang, W. (2000). "A model to determine the optimal critical level and the monitoring intervals in condition-based maintenance," International Journal of Production Research 38 (6), 1425-1436.

Yuniarto M. N. and Labib A. W. (2006). "Fuzzy adaptive preventive maintenance in a manufacturing control system: A step towards self-maintenance," International Journal of Production Research 44(1): 159-180.

Zhang, Y. L., Yam R. C. M. and Zuo Ming J. (2001). "Optimal replacement policy for a deteriorating production system with preventive maintenance." International Journal of Systems Science 32(10): 1193-1198. 